\title{
PL1 | The Cytomotive Switch In Actins And Tubulins
}

Löwe, Jan (Laboratory of Molecular Biology, Cambridge, GBR)

Protein filaments are used in cells of all sizes to position other molecules in time and space. These filaments can be called cytoskeletons, and they perform critical organising functions in a wide array of fundamental processes. The number of protein families used to form cytoskeletons is, perhaps, surprisingly small given the diversity of processes in which cytoskeletons play a role. In particular, in cytoskeletons across all domains of life, members of the actin and tubulin superfamily are found remarkably often, while the filaments they form are used in quite different processes. For example, prokaryotic actin and tubulin filaments such as ParM, FtsZ, MreB, TubZ, MamK, AlfA and BtubAB illustrate their functional diversity. One explanation for the structural but not functional conservation is that actin and tubulin proteins are special - that their structures encode hard-to-evolve mechanistic features that are useful in many contexts. Using a variety of structural bioinformatic analyses we integrated existing data about the tubulin superfamily to show that one conserved and (putatively) hard-to-evolve feature found across eukaryotic and bacterial tubulins is conformational switching upon polymerisation. We also showed that a similar feature is found across the actin superfamily. So far it is clear that conformational switching upon polymerisation mechanisms underlie the dynamics, and therefore functions, of microtubules and F-actin. Importantly, there are now several examples of the same conformational switching upon polymerisation feature being exploited in the functioning of prokaryotic actin and tubulin filaments as well: notably in the treadmilling FtsZ filament during bacterial cell division, and for the assembly of the magnetosome-positioning MamK filament (amongst other examples). Interestingly, actin MreB is an outlier in the sense that it does not seem to switch upon polymerisation, and this is in line with previous data that showed MreB filaments to be non-dynamic, being moved along by cell wall synthesis. Altogether, these findings suggest that conformational switching upon polymerisation is what makes both actin and tubulin special, and especially useful for building dynamic cytoskeletons using cytomotive filaments. 